\title{
Examining the readability of patient-informed consent forms
}

This article was published in the following Dove Press journal:

Open Access Journal of Clinical Trials

18 October 2010

Number of times this article has been viewed

\section{Marli Terblanche \\ Lesley Burgess \\ TREAD Research/Cardiology Unit, Department of Internal Medicine, Tygerberg Hospital and University of Stellenbosch, Parow, South Africa}

Correspondence: Marli Terblanche Room 4I, Department of Cardiology, 8th Floor, Tygerberg Hospital, Parow 7500 , South Africa

Tel +27219317825

$\mathrm{Fax}+27219333597$

Email marli@treadresearch.com
Primary objective: To investigate the readability of informed consent forms (ICF) used at TREAD Research, a private clinical trial research unit located in Tygerberg Hospital.

Secondary objective: To assess if there is a difference in readability between therapeutic areas, as well as a difference in readability over two time periods.

Methods: The readability of 84 ICFs given to patients at TREAD Research between the years 2000 and 2009 was quantitatively assessed by means of the Flesch-Kincaid Reading Ease, Flesch-Kincaid Grade Level, and Gunning-Fog index.

Results: The mean \pm standard deviation (SD) Flesch-Kincaid Reading Ease score for the 84 ICFs was $46.60 \pm 5.62$ (range 33.2-65.6). The mean \pm SD grade level was $12.13 \pm 1.8$ (range 8.3-14.9) using the Flesch-Kincaid formula and $13.96 \pm 1.22$ (range 10.3-16.6) using the Gunning-Fog index. Readability at grade level 8 was only found in $1.2 \%$ of all the ICFs assessed. No differences were found in readability between therapeutic areas or over the two time periods.

Conclusions: The main finding is that these forms are too complex to be understood by average study participants and their families.

Keywords: informed consent forms, readability, literacy

\section{Introduction}

Informed consent is an ongoing process in which a prospective participant is informed about the facts of a specific clinical trial so that he/she can decide whether or not to agree to participate or to continue to participate in a specific clinical trial. ${ }^{1}$ It involves signing a written consent form, which represents the basis of the person's willingness to participate in a trial.

Informed consent forms (ICF) often present highly complex information that must be understood by patients. It is important that subjects truly understand specific elements in the form, including the purpose of the study, the randomization process, and risks and benefits in participating, and that participation is voluntary. ${ }^{2}$ Complexity of the consent form has been shown to be a major barrier to comprehension for many patients. ${ }^{2}$ Examples of these barriers include excessive length of the form, inadequate time to read the consent, the reading level, and the format and layout of the form. These obstacles are especially problematic for subjects with low literacy skills.

About $25 \%$ of American adults are classified as having 'low literacy skills'. ${ }^{3}$ American literacy surveys estimate that at least 40 million adults are functionally illiterate (left school before grade 7) and another 50 million are only marginally literate. ${ }^{4}$ As a result of educational deprivation in the past, the extent of this problem in South Africa is even greater. According to the Project Literacy Web site, 
there are currently 4.7 million adults in South Africa who never went to school and are totally illiterate. ${ }^{4}$ A further 4.9 million adults are functionally illiterate, as they left school before grade $7 .{ }^{4}$

Studies reveal that ICFs used in general medical research are often written at a reading level that exceeds that of study participants, the general population, and the mandates of institutional review boards (IRB). ${ }^{5}$ Incomprehensible documents increase risks to both the patient and the health care providers/sponsors. They can lead to therapeutic misconceptions where subjects believe that they will receive the treatment best suited for them and often misunderstand the nature or purpose of the study or fail to retain the information they do understand.

Standard readability scores can be used to measure the readability of a document and thereby assess whether a specific ICF is at an understandable reading level. These scoring systems count the number of words in a sentence, the number of syllables in words, and/or the number of characters in a word or phrase. Specific formulae are then used, depending on the scoring system used, and a grade level or the number of years of education will be determined to give an indication of the readability of the document.

Ideally, the goal is to develop ICFs that are both short and written in simple and comprehensible language so that they facilitate a greater level of understanding and enable potential subjects to make truly informed decisions about research study participation.

\section{Objectives}

The primary objective is to investigate the readability of ICFs used at TREAD Research. Secondary objectives are to assess if there is a difference in readability between therapeutic areas as well as over two time periods.

\section{Methods}

This study was conducted by TREAD Research, a private clinical trial research unit located in Tygerberg Hospital, Parow, Western Cape. This unit focuses on sponsor-driven trials investigating chronic diseases of lifestyle and is affiliated to the Cardiology Unit of Stellenbosch University.

The readability of 84 ICFs used at TREAD Research over the past 10 years were quantitatively assessed by means of the Flesch-Kincaid Reading Ease, ${ }^{6}$ Flesch-Kincaid Grade Level, ${ }^{7}$ and Gunning-Fog index. ${ }^{8}$

These scoring systems give an indication of how easy a text is to read. The tool used to determine these scores is powered by the open-source project PHP Text Statistics and is available in the Web site http://www.addedbytes. com/code/readability-score/ Each ICF had been reviewed and approved by the Health Research Ethics Committee of Stellenbosch University.

\section{Flesch-Kincaid Reading Ease}

The Flesch-Kincaid readability tests are designed to indicate comprehension difficulty when reading a passage of contemporary English. There are two tests: the Flesch-Kincaid Reading Ease and the Flesch-Kincaid Grade Level. Both tests are measured by using word length as well as sentence length.

In the Flesch reading test, higher scores indicate material that is easier to read; lower numbers mark passages that are more difficult to read. The formula and interpretation for the Flesch Reading Ease Score are explained in Appendix A.

\section{Flesch-Kincaid Grade Level}

The Flesch-Kincaid Grade Level formula translates a 0-100 score to a United States grade level. It also indicates the number of years of education generally required to understand the text, relevant when the formula results in a number greater than 12. The formula and interpretation for the FleschKincaid Grade Level are explained in Appendix B.

\section{Gunning-Fog index}

The Gunning-Fog index is a measure of text readability based upon sentence length and difficult words in a passage. The underlying message is that short sentences written in plain English achieve a better score than long sentences written in complicated language. The ideal score for readability with the Gunning-Fog index is 7 or 8 . Anything above 12 is too hard for most people to read.

\section{Results}

The 84 ICFs included studies in six therapeutic areas, namely cardiovascular $(n=26)$, diabetes $(n=19)$, rheumatology $(n=12)$, hypercholesterolemia $(n=11)$, hypertension $(n=6)$, and others $(n=10)$. The 'others' category includes studies in the following therapeutic areas: aspergillosis, communityacquired pneumonia, neurology, obesity, nonsteroidal antiinflammatories, and oncology. The readability scores were done on each of these specific therapeutic areas.

Statistical differences in readability were tested between the different therapeutic areas using the analysis of variance test in Excel (Microsoft, Redmond, WA). Readability for ICFs approved in the years 2000-2005 and 2006-2009 were also compared using a $t$-test in Microsoft Excel. 
The mean \pm standard deviation (SD) Flesch-Kincaid Reading Ease score for the 84 ICFs was $46.60 \pm 5.62$ (range 33.2-65.6). The mean \pm SD grade level was $12.13 \pm 1.8$ (range 8.3-14.9) using the Flesch-Kincaid formula and $13.96 \pm 1.22$ (range 10.3-16.6) using the Gunning-Fog index. The mean scores are presented in Figure 1. The scoring using the Gunning-Fog index is slightly higher than those from the Flesch-Kincaid formula.

Readability on a grade level 8 was only found in $1.2 \%$ $(n=1)$ of all the ICFs assessed using the Flesch-Kincaid Grade Level formula and none using the Gunning-Fog index. Only one study was found to have a Flesch-Kincaid Reading Ease score of more than 60, which indicates a 'standard' reading level (Figure 2).

Readability for the ICFs was similar across all therapeutic areas, and no statistical difference was found between them (Table 1). In addition, no statistical difference was found in readability for studies approved in the years 2000-2005 and 2006-2009. The number of ICFs taken from each year is shown in Table 2.

\section{Discussion}

This study examined the ICFs of 84 studies used in the last 10 years at TREAD Research. The main finding of this study is that these forms are too complex to be understood by the average study participant and his/her family.

The ICF of only one study was above the grade level of 65 , which indicates a 'standard' readability score. On average,

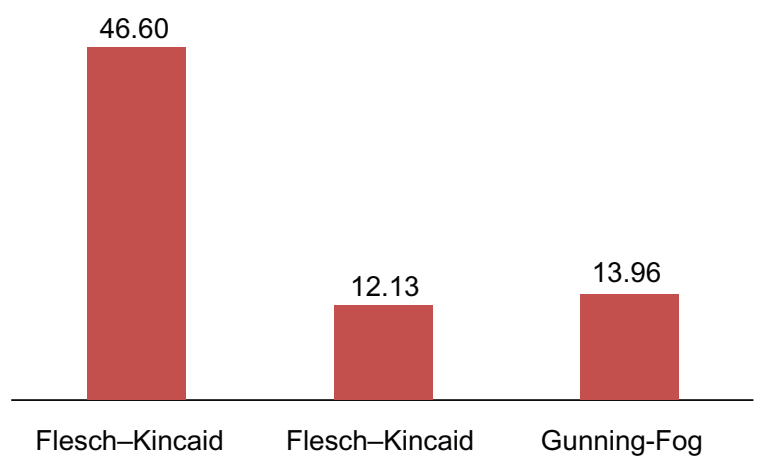

Figure I Mean readability scores of all studies $(n=84)$ using three different formulae.

consent forms of most of the studies scored between 45 and 50 , which indicates 'fairly difficult' to 'difficult' reading standards.

According to a previous study done at our unit, $46.2 \%$ of our patient population had completed grade 8 or less. ${ }^{9}$ It, therefore, appears that the readability of ICFs is clearly above the education level of our average patient population.

It is essential that ICFs are written in clear, direct language to ensure comprehension. Words longer than three syllables, long sentences, passive sentences, and medical vocabulary are among many factors affecting the readability standards. ${ }^{10}$ Strategies to simplify language include using short, familiar words or simple synonyms; limiting the use of polysyllabic words; and keeping sentence length less than 12 words and paragraph length less than 7 lines. ${ }^{10}$

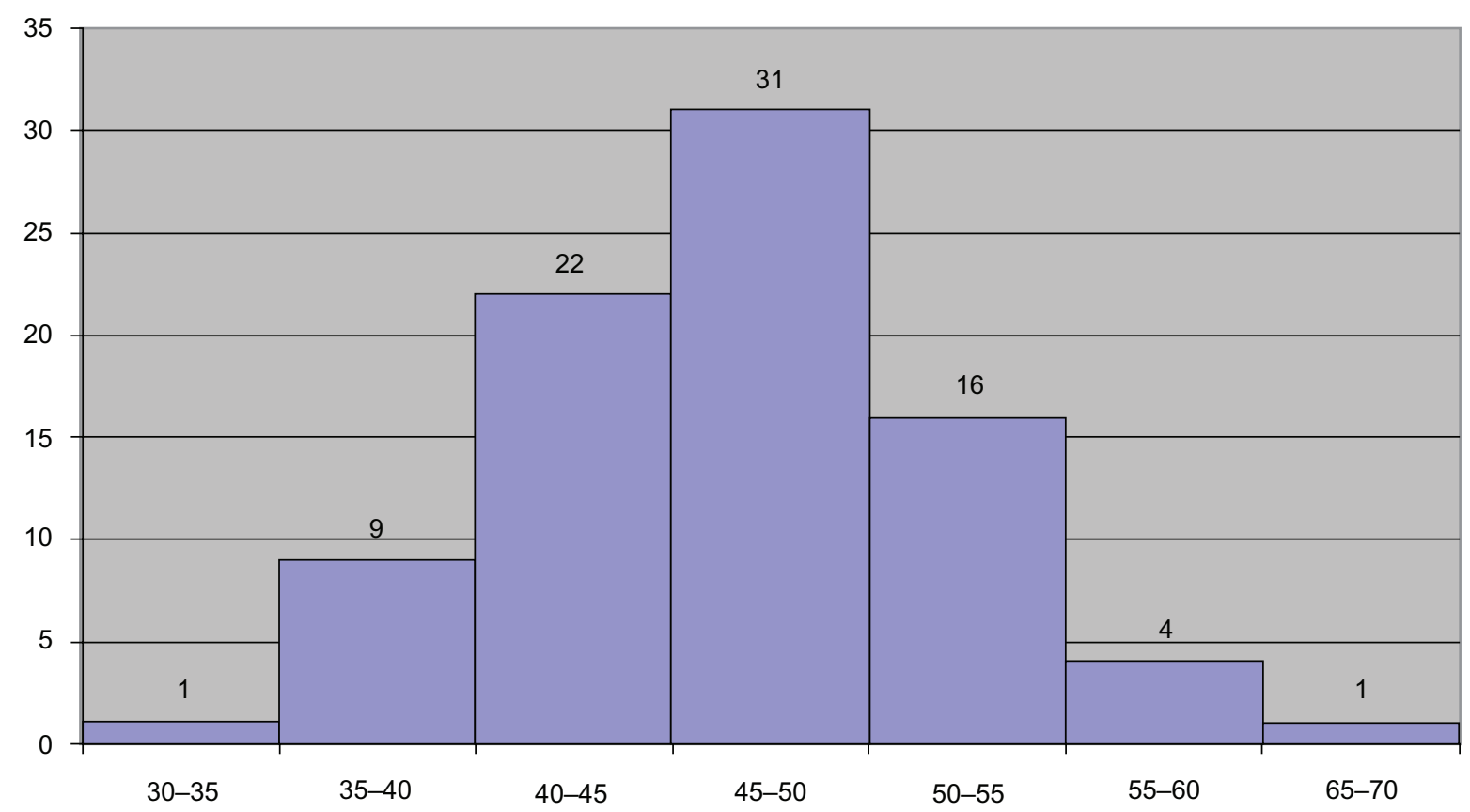

Figure 2 Distribution of studies using the Flesch-Kincaid Reading Ease score. 
Table I Mean readability scores across all therapeutic areas

\begin{tabular}{lllllll}
\hline & $\begin{array}{l}\text { Flesch-Kincaid } \\
\text { Reading ease }\end{array}$ & $\begin{array}{l}\text { Flesch-Kincaid } \\
\text { Grade level }\end{array}$ & $\begin{array}{l}\text { Gunning-Fog } \\
\text { index }\end{array}$ & No. of words & $\begin{array}{l}\text { Words/ } \\
\text { sentence }\end{array}$ & $\begin{array}{l}\text { Syllables/ } \\
\text { word }\end{array}$ \\
\hline CVS & 46.44 & 12.03 & 13.75 & 3774 & 21.06 & 1.64 \\
Diabetes & 47.80 & 12.04 & 13.87 & 5454 & 21.96 & 1.62 \\
Rheumatology & 48.11 & 11.81 & 13.83 & 5513 & 21.13 & 1.62 \\
Cholesterol & 48.57 & 11.74 & 13.60 & 4672 & 21.17 & 1.62 \\
HPT & 43.65 & 12.68 & 14.58 & 4589 & 22.14 & 1.66 \\
Others & 42.56 & 13.09 & 14.85 & 4067 & 23.19 & 1.66 \\
\hline
\end{tabular}

Abbreviations: CVS, computer vision syndrome; HPT, home pregnancy test.

The main focus of an ICF is to protect patients from unfairness and exploitation. However, recently, this focus appears to have shifted to facilitate patients to be able to make more independent decisions regarding their medical care or whether to become involved in medical research. ${ }^{11}$ The ICFs have had to change from being merely a list of information to clearly written text that is readable and understandable by most patients and family members. In 1988, Minnesota enacted a law requiring that documents such as ICFs be written at a 7.5-grade reading level to ensure that patients understand the therapeutic options available to them. ${ }^{11}$

The ultimate goal is to develop ICFs that will be understood by the general population participating in clinical research. The legal and scientific aspects of an ICF greatly complicate this set goal. There are, however, ways to improve readability and comprehension other than simplifying reading standards:

1. Give potential participants more time to carefully read the ICF, as the length of ICFs has steadily increased over time and it is often found that lengthy ICFs go hand in hand with more complex documents and studies involving greater risk. ${ }^{10}$

2. Eliminate factors that complicate ICFs, such as the length of the document, use shorter sentences and paragraphs, use less polysyllabic and unfamiliar words, and omit information that is regarded as irrelevant.

3. Change in formatting and making layout modifications have also proved beneficial to enhance comprehension. In a study by Hochhauser, it was noted that 'document design and layout are at least as important, perhaps more important' than reading level estimates. ${ }^{12}$ By incorporating changes such as using bigger fonts, no single spacing, clear headings, and improving the overall design and

Table 2 Number of informed consent forms (ICFs) taken each year Year 2000200120022003200420052006200720082009 \begin{tabular}{lllllllllll}
\hline ICF & I & 4 & 9 & 9 & 4 & 13 & 9 & 14 & 8 & 13
\end{tabular} organization of the ICFs, one can hope to assist in the understanding.

4. Recent studies demonstrate that the performance of documents, like rewriting, redesigning, and the addition of graphics, resulted in better understanding of the information included in the ICF than the use of a readability formula alone..$^{13}$

\section{Conclusion}

ICFs are too complex to be understood by average study participants and their families. The goal is to develop ICFs that will be understood by the general population participating in clinical research. There are many obstacles in reaching these goals. Most important is the financial cost of implementing changes to ICFs globally. Standardizing ICFs and the informed consent process should be prioritized. First, comprehension could be enhanced by developing templates of the documents and thus including more important and less irrelevant information and by using a standardized process. Second, by providing the required education to protocol and consent writers, as well as investigators and members of the institutional review boards, the readability and comprehension of the general population participating in clinical research can be enhanced.

\section{Disclosure}

The authors report no conflicts of interest in this work.

\section{References}

1. South African Department of Health. South African National Clinical Trial Register. Available at: http://www.sanctr.gov.za/whatisinformedconsent/tabid/ 191/Default.aspx. Accessed 2009 Nov.

2. South African Department of Health. Guidelines for Good Practice in the Conduct of Clinical Trials with Human Participants in South Africa. 2nd ed. Pretoria, South Africa: Department of Health; 2006.

3. Kirsch IS, Jungeblut A, Jenkins L, Kolstad A. Adult Literacy in America: A First Look at the Results of the National Adult Literacy Survey. Washington (DC): Office of Education Research and Improvement, Department of Education; 1993.

4. Project Literacy. Leaders in adult learning. Available at: http://www. projectliteracy.org.za/. Accessed 2009 Dec. 
5. Hochhauser M. Informed consent: reading and understanding are not the same. Applied Clinical Trials Online. 2004 Apr 1. Available at: http:// www.appliedclinicaltrialsonline.com. Accessed 2010 Mar 3.

6. The Flesch Reading Ease Readability Formula. Available at: http://www.readabilityformulas.com/flesch-reading-ease-readabilityformula.php. Accessed 2010 Aug 10.

7. The Flesch Grade Level Readability Formula. Available at: http://www.readabilityfor mulas.com/flesch-grade-level-readabilityformula.php. Accessed 2010 Aug 10.

8. The Gunning's Fog Index (or FOG) Readability Formula. Available at: http://www.readabilityformulas.com/gunning-fog-readability-formula. php. Accessed 2010 Aug 10.

9. Burgess LJ, Sulzer NU, Emanuel S. Clinical trial remuneration: the patients' perspective. S Afr Med J. 2008;98(2):95-97.
10. Wittenberg KM, Dickler HB. Universal use of short and readable informed consent documents: How do we get there? Creating Informed Consent documents that inform: A Literature Review. Association of American Medical Colleges Appendix C, Feb 2007. Available at: http:// www.aamc.org/research/clinicalresearch/hdickler-mtgsumrpt53007. pdf. Accessed 2010 Aug 10.

11. Grossman SA, Piantadosi S, Covahey C. Are informed consent forms that describe clinical oncology research protocols readable by most patients and their families? J Clin Oncol. 1994;12(10):2211-2215.

12. Hochhauser M. Some overlooked aspects of consent form readability. IRB. 1997;19(5):5-9.

13. Knapp P, Raynor DK, Silcock J, Parkinson B. Performance-based readability testing of participant information for a Phase 3 IVF trial. Open Access http://www.trialsjournal.com/content/10/1/79. Accessed 2010 Aug 11. 


\section{Appendix A}

Formula for the Flesch Reading Ease Score (FRES):

$206.835-1.015\left(\frac{\text { total words }}{\text { total sentences }}\right)-84.6\left(\frac{\text { total syllables }}{\text { total words }}\right)$.

Scores can be interpreted as shown in the table below:

\begin{tabular}{ll}
\hline Score & Notes \\
\hline $90.0-100.0$ & $\begin{array}{l}\text { Easily understandable by an average II-year-old } \\
\text { student }\end{array}$ \\
$60.0-70.0$ & $\begin{array}{l}\text { Easily understandable by 13- to I5-year-old student } \\
0.0-30.0\end{array}$ \\
\hline
\end{tabular}

According to Readability Formulas.com, a score between 60 and 70 is largely considered acceptable. The following table is also helpful to assess the ease of readability in a document:

\begin{tabular}{ll}
\hline $90-100$ & Very easy \\
$80-89$ & Easy \\
$70-79$ & Fairly easy \\
$60-69$ & Standard \\
$50-59$ & Fairly difficult \\
$30-49$ & Difficult \\
$0-29$ & Very confusing \\
\hline
\end{tabular}

\section{Appendix B}

The Flesch-Kincaid Grade Level formula:

$$
0.39\left(\frac{\text { total words }}{\text { total sentences }}\right)+11.8\left(\frac{\text { total syllables }}{\text { total words }}\right)-15.59 \text {. }
$$

The result is a number that corresponds with a grade level. For example, a score of 8.2 would indicate that the text is expected to be understandable by an average student in eighth grade (usually around ages 13-14 years in the United States of America).
Open Access Journal of Clinical Trials

\section{Publish your work in this journal}

The Open Access Journal of Clinical Trials is an international, peerreviewed, open access journal publishing original research, reports, editorials, reviews and commentaries on all aspects of clinical trial design, management, legal, ethical and regulatory issues, case record form design, data collection, quality assurance and data auditing

\section{Dovepress}

methodologies. The manuscript management system is completely online and includes a very quick and fair peer-review system, which is all easy to use. Visit http://www.dovepress.com/testimonials.php to read real quotes from published authors. 